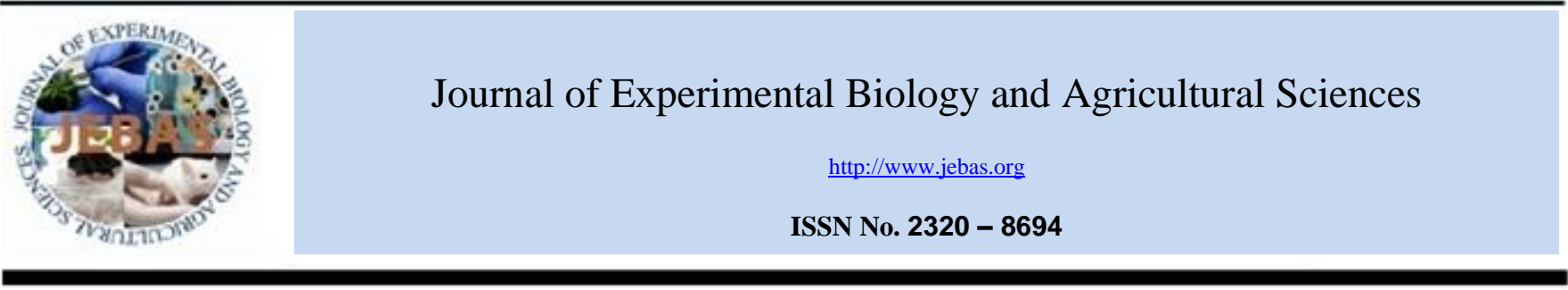

\title{
EFFECT OF BENZYLADENINE AND SALICYLIC ACID ON BIOCHEMICAL TRAITS OF TWO CULTIVARS OF CARNATION
}

\author{
Anita Ramtin ${ }^{1}$, Sepideh Kalatejari ${ }^{1, *}$, Roohangiz Naderi ${ }^{2}$ and Mohammad Matinizadeh ${ }^{3}$ \\ ${ }^{1}$ Department of Horticulture, Science and Research branch, Islamic Azad University, Tehran, Iran \\ ${ }^{2}$ Department of Horticultural Sciences, Faculty of Agricultural Sciences and Natural Resources, University of Tehran, Karaj, Iran \\ ${ }^{3}$ Department of Forest, Research Institute of Forest and Rangelands, Tehran, Iran
}

Received - February 11, 2016; Revision - April 30, 2016; Accepted - May 12, 2016

Available Online - June 30, 2016

DOI: http://dx.doi.org/10.18006/2016.4(4).427.434

KEYWORDS
Carnation
Benzyladenine
Salicylic Acid
Spraying
Biochemical traits

\begin{abstract}
Plant growth regulators have significant effect on the delaying senescence in buds and flowers. Present study was conducted to find out the effects of Salicylic acid and Benzyl adenine spraying on various biochemical traits such as membrane stability index, chlorophyll a, and b content, total chlorophyll content, sugar content, catalase, and peroxidase enzymes activity, and proline content in Carnation. Study was conducted in a split plot design with three replications and various concentrations of hormones, cultivars of carnation and measurement time was used as a factor of interaction. Results of study showed that all the traits were significantly affected by spraying of these tow hormones. Cultivar factor was most effective in chlorophyll a, b, and total chlorophyll content, catalase enzyme activity, proline content and sugar content. Among these two hormones, spraying of Salicylic acid @ $100 \mu \mathrm{M}$ was found most effective treatment. Further, time of measurements and cultivar factors were also found effective in all of traits.
\end{abstract}

* Corresponding author

E-mail: azad.sciences@gmail.com (Sepideh Kalatejari)

Peer review under responsibility of Journal of Experimental Biology and Agricultural Sciences.

Production and Hosting by Horizon Publisher India [HPI] (http://www.horizonpublisherindia.in/).

All rights reserved.
All the article published by Journal of Experimental Biology and Agricultural Sciences is licensed under a Creative Commons Attribution-NonCommercial 4.0 International License Based on a work at www.jebas.org.

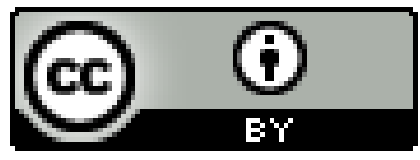




\section{Introduction}

Carnation (Dianthus caryophyllus L.) is one of the most popular ornamental flower and it is well known throughout the world for its beauty, diversity of colors, excellent keeping quality, and wide flower range (Kharrazi et al. 2011). It belongs to family Caryophyllaceae and has more than 300 species (Jurgens et al., 2003). Members of the genus Dianthus are fairly diverse, as their origins range from Southern Russia to Alpine Greece and the Auvergne mountains of France. The Dianthus species are adapted to the cooler Alpine regions of Europe and Asia, and are also found in Mediterranean coastal regions (Australian Government 2006). The genus Dianthus contains both annual and perennial caespitose plants; among these some of its species are cultivated for hundreds of years for ornamental purposes. Modern cut-flower varieties of carnation have been selected for its flower size, petal number, stem length, and disease resistance. Since 19th century, it is extensively produced for commercial purpose under field and green house conditions. After transferring germplasm to USA, carnation breeding and growing for the cut-flower market became very popular in the USA (OGTR 2006).

Regulation of the growth of ornamental plants for commercial purposes is a vital aspect in the ornamental production. Both practical requirements and aesthetic aspect must be considering in order supplying high quality product for buyers (John Carey \& Mark 2008). According to Arias \& López (2010), method used for commercial production should always favor the improvement in visual (size, compactness, branching, color, etc.) and physiological quality (resistance to stresses, leaving the rest, improve the postharvest, etc.) of carnation. Breeding of carnation is not an easy task because of flower texture, its flowers are normally protandrous and outcross occurred typically (Behroozian et al. 2013). Further, stem and leaf tissues of carnation plants also contain natural antisenescence factors which is also an obstacle in the large scale production (Isinger 1977). Now in these days, commercial flower producer used various plant growth regulators for increasing delayed senescence of buds, flowers, or leaves (John Carey \& Mark 2008).

The cytokinins are plant specific phytohormones (Bubán, 2000) which play a central role in the regulation of cell cycle and numerous developmental processes. These are present in all plant tissues but are abundant in root tip, shoot apex and immature seeds (Schmülling, 2004). Cytokinins are well known to defer leaf senescence in carnation and rose flowers. The main group of synthetic cytokinins includes N6 substituted adenines like kinetin (6-furfurylaminopurine), benzyl adenine (6-benzylaminopurine, $\quad$ BA), 6-benzylamino-9(tetrahydropyran-2-yl) -9H- purine; PBA (Bubán, 2000). Recently, a synthetic cytokinin benzyl adenine (BA) has been released in the U.S. as configure for use on ornamental plants (John Carey \& Mark 2008). It elicits the plant growth and development responses, setting blossoms and stimulating fruit richness by stimulating cell division. Lately, numerous postharvest technologies have been established for enhancing postharvest of fresh and perishable products including fruit, vegetables and cut flowers.

Salicylic acid is a messenger molecule regulating developmental procedures and it control the biotic and abiotic stresses (Zarghami et al. 2014). It also inhibiting ethylene biosynthesis and delaying fruit senescence (Kademi \& Ershadi 2013). Further, responses of environmental stresses in plants could be modulated by salicylic acid signaling (Yao \& Tian, 2004; Supapvanich \& Promyou, 2013; Ramtin et al., 2015). The purpose of this experiment was to study effects of these plants growth regulators spray on important biochemical characteristics of two carnations cultivars with time concern.

\section{Materials and methods}

Study was conducted under greenhouse condition at the in Tehran, Iran. The experimental design was complete randomized design (CRD) in a split plot design with three replications. Various concentration of growth regulators (SA $50 \mu \mathrm{M}$, SA $100 \mu \mathrm{M}$, SA $200 \mu \mathrm{M}$, BA $50 \mu \mathrm{M}$, BA $100 \mu \mathrm{M}$, BA150 $\mu \mathrm{M}$, and Control), different cultivars (Spray, and Standard), and three measured times (3 days after harvest, 6 days after harvest, and 9 day after harvest) was taken as a factor of interaction, indicating change of important enzymes and substances in cut carnation during postharvest. Spraying on plants were two times, first spraying was before bud opening and another spraying was three days before harvest. Membrane stability index were determined by method described by Sairam (1994), while chlorophyll a, chlorophyll b and total chlorophyll content was determined by the method given by Arnon (1967) with some modification. Further, sugar content by Dey (1990), catalase, and peroxidase enzymes activity by Rani et al. (2004), and proline content was estimated by Bates et al. (1973).

\section{Results}

\subsection{Treatment factor}

According to ANOVA (table 1), all the studied traits were significantly affected by spraying various concentrations of plant growth hormones $(\mathrm{p} \leq 0.01)$. Further, time measurements factor was also effective on all the studied traits $(\mathrm{p} \leq 0.01)$. Similarly, cultivar factor such as chlorophyll $a, b$, and total chlorophyll content, catalase enzyme activity, proline content $(p \leq 0.01)$ and sugar content $(p \leq 0.05)$ was also effective on. Among various concentrations, $100 \mu \mathrm{M}$ concentration of salicylic acid caused highest stability in membrane while control plants had the lowest stability. A significant effect of benzyl adenine spray was reported on the chlorophyll content and highest total chlorophyll content was related to $100 \mu \mathrm{M}$ spray of benzyl adenine in return while control plants had the lowest total chlorophyll content. 
Table 1 Variance of analysis results for three studied factors.

\begin{tabular}{|c|c|c|c|c|c|c|c|c|c|}
\hline \multirow[t]{2}{*}{ SOV } & \multirow[t]{2}{*}{ df } & \multicolumn{8}{|c|}{ Mean of square } \\
\hline & & MSI & TChl & Chl a & $\mathrm{Chl} \mathrm{b}$ & TSS & CAT & POD & Pro \\
\hline Rep & 2 & 33.489 & $7.0 \mathrm{E}-7$ & $2.5 \mathrm{E}-7$ & 4.4E-8 & $5.9 \mathrm{E}-9$ & 0.0005 & 0.0041 & 5.248 \\
\hline HS & 6 & $1114.3^{* *}$ & $2.1 \mathrm{E}-6^{* *}$ & $7.3 \mathrm{E}-7^{* *}$ & $1.2 \mathrm{E}-7^{* *}$ & $1.3 \mathrm{E}-7^{* *}$ & $0.002^{* *}$ & $0.004^{* *}$ & $16.7^{* *}$ \\
\hline HS error & 12 & 26.41 & 2.2E-9 & $8.2 \mathrm{E}-9$ & $1.0 \mathrm{E}-9$ & $8.0 \mathrm{E}-10$ & $1.5 \mathrm{E}-5$ & 0.0003 & 0.442 \\
\hline MT & 2 & $4647.3^{* *}$ & $2.2 \mathrm{E}-6^{* *}$ & $2.8 \mathrm{E}-7^{* *}$ & $4.0 \mathrm{E}-7^{* *}$ & $7.4 \mathrm{E}-7^{* *}$ & $7.6 \mathrm{E}-5^{* *}$ & $0.0007^{* *}$ & $1.86^{* *}$ \\
\hline MT*HS & 12 & $28.7^{* * *}$ & $1.4 \mathrm{E}-8^{* *}$ & $2.3 \mathrm{E}-8^{* *}$ & $3.5 \mathrm{E}-9^{* *}$ & $2.2 \mathrm{E}-9^{*}$ & $5.1 \mathrm{E}-5^{* *}$ & $4.6 \mathrm{E}-5^{\mathrm{ns}}$ & $1.98^{* * *}$ \\
\hline MT error & 4 & 5.075 & $3.0 \mathrm{E}-8$ & $1.4 \mathrm{E}-8$ & $2.2 \mathrm{E}-9$ & $3.5 \mathrm{E}-9$ & 0.00004 & 0.00015 & 0.828 \\
\hline CV & 1 & $42.21^{*}$ & $1.1 \mathrm{E}-6^{* *}$ & $5.1 \mathrm{E}-7^{* *}$ & $5.2 \mathrm{E}-7^{* *}$ & $2.7 \mathrm{E}-7^{* *}$ & $0.00056^{* *}$ & $0.007045^{* *}$ & $0.933^{*}$ \\
\hline $\mathrm{HS} * \mathrm{CV}$ & 6 & $294.56^{* *}$ & $8.8 \mathrm{E}-8^{* *}$ & $3.4 \mathrm{E}-8^{* *}$ & $1.3 \mathrm{E}-8^{* *}$ & $1.4 \mathrm{E}-8^{* *}$ & $0.00035^{* *}$ & $0.00045^{* *}$ & $1.004^{*}$ \\
\hline $\mathrm{MT}^{*} \mathrm{CV}$ & 2 & $879.01^{* *}$ & $1.0 \mathrm{E}-6^{* *}$ & $4.1 \mathrm{E}-7^{* *}$ & $5.2 \mathrm{E}-8^{* *}$ & $3.2 \mathrm{E}-8^{* *}$ & $0.0001^{* *}$ & $0.0007^{* *}$ & $2.494^{* *}$ \\
\hline MT*HS*CV & 12 & $8.76^{\mathrm{ns}}$ & $1.5 \mathrm{E}-8^{* *}$ & $3.2 \mathrm{E}-8^{* *}$ & $3.2 \mathrm{E}-9^{* *}$ & $5.8 \mathrm{E}-9^{* *}$ & $0.00003^{* *}$ & $0.00002^{\mathrm{ns}}$ & $0.448^{* *}$ \\
\hline CV error & 2 & 249.86 & $9.9 \mathrm{E}-7$ & $3.8 \mathrm{E}-7$ & $4.7 \mathrm{E}-8$ & $4.9 \mathrm{E}-9$ & 0.0014 & 0.00008 & 0.0564 \\
\hline Total & 64 & 6.86 & 4. $9 \mathrm{E}-9$ & 5.3E-9 & $9.6 \mathrm{E}-10$ & $1.1 \mathrm{E}-9$ & $9.7 \mathrm{E}-6$ & 0.00004 & 0.1589 \\
\hline
\end{tabular}

* Significant at $P \leq 0.05 ; * *$, Significant at $P \leq 0.01$, ns = non-significant; here Membrane stability index (MSI), Total chlorophyll content (TChl), chlorophyll a content (Chl a), chlorophyll b content (Chl b), Total soluble sugar (TSS), Catalase activity (CAT), Peroxidase Activity (POD), Proline content (Pro), Hormone spraying (HS), Measured time (MT), Cultivar (CV).

Table 2 Effect of the various hormone concentrations on the various studied characteristics of carnation.

\begin{tabular}{|c|c|c|c|c|c|c|c|c|}
\hline & MSI & TChl & Chl a & Chl b & TSS & CAT & POD & Pro \\
\hline SA $50 \mu M$ & $53.027^{\mathrm{a}}$ & $0.00166^{\mathrm{c}}$ & $0.000829^{c}$ & $0.000424^{\mathrm{d}}$ & $0.00074^{\mathrm{a}}$ & $0.0297^{\mathrm{b}}$ & $0.0459^{\mathrm{bc}}$ & $4.1417^{\mathrm{b}}$ \\
\hline SA $100 \mu M$ & $56.563^{\mathrm{a}}$ & $0.00187^{\mathrm{b}}$ & $0.001019^{\mathrm{a}}$ & $0.000538^{\mathrm{a}}$ & $0.00073^{\mathrm{a}}$ & $0.0474^{\mathrm{a}}$ & $0.07365^{\mathrm{a}}$ & $4.524^{\mathrm{a}}$ \\
\hline SA $200 \mu M$ & $45.715^{\mathrm{c}}$ & $0.00124^{\mathrm{e}}$ & $0.000613^{\mathrm{d}}$ & $0.000371^{\mathrm{e}}$ & $0.000597^{\mathrm{c}}$ & $0.0200^{\mathrm{c}}$ & $0.0491^{\mathrm{b}}$ & $4.2512^{b}$ \\
\hline BA $50 \mu \mathrm{M}$ & $44.594^{\mathrm{c}}$ & $0.00152^{\mathrm{d}}$ & $0.000816^{\mathrm{c}}$ & $0.000445^{\mathrm{c}}$ & $0.000628^{\mathrm{b}}$ & $0.0232^{\mathrm{c}}$ & $0.0369^{\mathrm{d}}$ & $3.2167^{\circ}$ \\
\hline BA $100 \mu \mathrm{M}$ & $49.795^{b}$ & $0.00192^{\mathrm{a}}$ & $0.000961^{\mathrm{b}}$ & $0.000514^{\mathrm{b}}$ & $0.000617^{b c}$ & $0.03122^{b}$ & $0.0435^{\mathrm{c}}$ & $3.7368^{\circ}$ \\
\hline BA $150 \mu \mathrm{M}$ & $38.923^{\mathrm{d}}$ & $0.00127^{\mathrm{e}}$ & $0.000625^{\mathrm{d}}$ & $0.000385^{\mathrm{e}}$ & $0.000564^{\mathrm{d}}$ & $0.0176^{\mathrm{d}}$ & $0.03147^{\mathrm{d}}$ & $2.4952^{\circ}$ \\
\hline Control & $34.001^{\mathrm{e}}$ & $0.00102^{f}$ & $0.000461^{\mathrm{e}}$ & $0.00031^{\mathrm{f}}$ & $0.00051^{\mathrm{e}}$ & $0.0135^{\mathrm{e}}$ & $0.02750^{\mathrm{e}}$ & $1.9527^{\mathrm{f}}$ \\
\hline
\end{tabular}

Means in a column followed by the same letter are not significantly different at $5 \%$ level; results are the mean of three replicates

Table 3 Effect of the time periods on the various growth characteristics of carnation.

\begin{tabular}{|lcccccccc|}
\hline & MSI & TChl & Chl a & Chl b & TSS & CAT & POD & Pro \\
\hline $\mathbf{1}^{\text {st }}$ Time & $57.532^{\mathrm{a}}$ & $0.00172^{\mathrm{a}}$ & $0.0009^{\mathrm{a}}$ & $0.000497^{\mathrm{a}}$ & $0.000732^{\mathrm{a}}$ & $0.0279^{\mathrm{a}}$ & $0.0484^{\mathrm{a}}$ & $3.66779^{\mathrm{a}}$ \\
\hline $\mathbf{2}^{\text {nd }}$ Time & $43.894^{\mathrm{b}}$ & $0.0015^{\mathrm{b}}$ & $0.00073^{\mathrm{b}}$ & $0.000466^{\mathrm{b}}$ & $0.000669^{\mathrm{b}}$ & $0.0256^{\mathrm{b}}$ & $0.0433^{\mathrm{b}}$ & $3.50412^{\mathrm{a}}$ \\
\hline $\mathbf{3}^{\text {rd }}$ Time & $36.84^{\mathrm{c}}$ & $0.00126^{\mathrm{c}}$ & $0.0007^{\mathrm{b}}$ & $0.000316^{\mathrm{c}}$ & $0.000477^{\mathrm{c}}$ & $0.0255^{\mathrm{b}}$ & $0.0402^{\mathrm{c}}$ & $3.25019^{\mathrm{b}}$ \\
\hline
\end{tabular}

Means in a column followed by the same letter are not significantly different at 5\% level, results are the mean of three replicates.

Also chlorophyll a, b content under the influence of $100 \mu \mathrm{M}$ salicylic acid, increased to the highest value although the lowest content of chlorophyll a, b was related to control. Spraying with salicylic acid improved sugar content. The highest value of sugar content was gained by Salicylic acid 50, $100 \mu \mathrm{M}$ treatments. Salicylic acid enhanced catalase enzyme activity that the most catalase activity was gained by spraying salicylic acid $100 \mu \mathrm{M}$. Similarly, results of peroxidase enzyme activity revealed that the enzyme activity was increased by spraying salicylic acid and the highest activity was related to spray of $100 \mu \mathrm{m}$ salicylic acid while the least activity was caused by spraying benzyl adenine $150 \mu \mathrm{M}$, and control. Also spraying salicylic acid $100 \mu \mathrm{M}$ raised the value of proline content (table 2).

\subsection{Time factor}

Results of time factor study revealed that value of various studied factors changes with the time $(\mathrm{p} \leq 0.01)$ (table 1$)$ and this value was decreased with the passing of time (table 3 ). 
Table 4 Mean comparison of carnation cultivars and its effects on various characters.

\begin{tabular}{|lcccccccc|} 
& MSI & TChl & Chl a & Chl b & TSS & CAT & POD & Pro \\
\hline Spray & $45.5097^{\mathrm{b}}$ & $0.001405^{\mathrm{b}}$ & $0.00082^{\mathrm{a}}$ & $0.00036^{\mathrm{b}}$ & $0.00058^{\mathrm{b}}$ & $0.02424^{\mathrm{b}}$ & $0.03652^{\mathrm{b}}$ & $3.38797^{\mathrm{b}}$ \\
\hline Standard & $46.6673^{\mathrm{a}}$ & $0.00159^{\mathrm{a}}$ & $0.00069^{\mathrm{b}}$ & $0.00049^{\mathrm{a}}$ & $0.00067^{\mathrm{a}}$ & $0.02848^{\mathrm{a}}$ & $0.05148^{\mathrm{a}}$ & $3.5601^{\mathrm{a}}$ \\
\hline
\end{tabular}

Means in a column followed by the same letter are not significantly different at $5 \%$ level; results are the mean of three replicates.

Table 5 Effect of the double interaction between hormone*cultivar on various studied traits of carnation.

\begin{tabular}{|c|c|c|c|c|c|c|c|c|}
\hline $\begin{array}{l}\text { Double interaction } \\
\text { (Hormone* Cultivar) }\end{array}$ & MSI & TChl & Chla & Chlb & TSS & CAT & POD & Pro \\
\hline SA50 $\mu \mathrm{M} *$ spray & $48.858^{\mathrm{c}}$ & $0.00154^{\mathrm{e}}$ & $0.000729^{\mathrm{e}}$ & $0.000465^{\mathrm{t}}$ & $0.000808^{\mathrm{a}}$ & $0.029474^{b}$ & $0.041074^{\mathrm{e}}$ & 4.197423 \\
\hline SA50 $\mu \mathrm{M} *$ standard & $57.197^{b}$ & $0.00179^{\mathrm{c}}$ & $0.000929^{c}$ & $0.000382^{\mathrm{c}}$ & $0.000668^{\mathrm{b}}$ & $0.02996^{\mathrm{b}}$ & $0.050746^{\mathrm{d}}$ & $4.085926^{\mathrm{bc}}$ \\
\hline SA100 $\mu \mathrm{M} *$ spray & $50.609^{\mathrm{c}}$ & $0.00174^{\mathrm{c}}$ & $0.000927^{\mathrm{c}}$ & $0.000516^{\mathrm{ab}}$ & $0.00064^{\mathrm{bc}}$ & $0.047305^{\mathrm{a}}$ & $0.064623^{\mathrm{b}}$ & $4.334373^{\mathrm{b}}$ \\
\hline SA100 $\mu \mathrm{M} *$ standard & $62.518^{\mathrm{a}}$ & $0.00202^{\mathrm{a}}$ & $0.001112^{\mathrm{a}}$ & $0.000561^{\text {ab }}$ & $0.000822^{\mathrm{a}}$ & $0.047428^{\mathrm{a}}$ & $0.082686^{\mathrm{a}}$ & $4.71358^{\mathrm{a}}$ \\
\hline SA200 $\mu M$ * spray & $47.642^{\mathrm{cd}}$ & $0.00120^{\mathrm{g}}$ & $0.000500^{\mathrm{g}}$ & $0.000304^{\mathrm{d}}$ & $0.000564^{\mathrm{c}}$ & $0.022577^{\mathrm{c}}$ & $0.032873^{\mathrm{f}}$ & $3.947815^{\mathrm{bc}}$ \\
\hline SA200 $\mu \mathrm{M} *$ standard & $43.789^{d}$ & $0.00129^{\mathrm{g}}$ & $0.000726^{\mathrm{e}}$ & $0.000439^{\mathrm{b}}$ & $0.000632^{\mathrm{bc}}$ & $0.021379^{\mathrm{c}}$ & $0.065262^{\mathrm{b}}$ & $4.554606^{\mathrm{ab}}$ \\
\hline BA50 $\mu \mathrm{M} *$ spray & $42.552^{\mathrm{d}}$ & $0.00138^{f}$ & $0.000788^{\mathrm{e}}$ & $0.000357^{\mathrm{c}}$ & $0.000589^{\mathrm{c}}$ & $0.021529^{\mathrm{c}}$ & $0.033678^{\mathrm{f}}$ & $3.218254^{\mathrm{c}}$ \\
\hline BA50 $\mu \mathrm{M} *$ standard & $46.636^{\mathrm{cd}}$ & $0.00166^{\mathrm{d}}$ & $0.000841^{\mathrm{d}}$ & $0.000533^{\mathrm{ab}}$ & $0.000668^{\mathrm{b}}$ & $0.024826^{\mathrm{bc}}$ & $0.040101^{\mathrm{e}}$ & $3.215047^{\mathrm{c}}$ \\
\hline 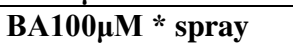 & $47.766^{\mathrm{cd}}$ & $0.00189^{\mathrm{b}}$ & $0.000876^{\mathrm{d}}$ & $0.000586^{\mathrm{a}}$ & $0.000564^{\mathrm{c}}$ & $0.019314^{\mathrm{d}}$ & $0.031779^{\mathrm{f}}$ & $3.306853^{\mathrm{c}}$ \\
\hline BA100 $\mu \mathrm{M} *$ standard & $51.826^{\mathrm{c}}$ & $0.00196^{\mathrm{a}}$ & $0.001046^{\mathrm{b}}$ & $0.000443^{\mathrm{b}}$ & $0.000670^{\mathrm{b}}$ & $0.043129^{\mathrm{a}}$ & $0.055149^{\mathrm{c}}$ & $4.166836^{\mathrm{b}}$ \\
\hline BA150 $\mu \mathrm{M}$ * spray & $36.307^{\mathrm{e}}$ & $0.00110^{\mathrm{h}}$ & $0.000635^{\mathrm{f}}$ & $0.000284^{\mathrm{d}}$ & $0.000536^{\mathrm{c}}$ & $0.016115^{\mathrm{e}}$ & $0.027472^{\mathrm{g}}$ & $2.760892^{\mathrm{d}}$ \\
\hline BA150 $\mu \mathrm{M} *$ standard & $41.539^{\mathrm{d}}$ & $0.00144^{\mathrm{f}}$ & $0.000614^{\mathrm{f}}$ & $0.000487^{\mathrm{b}}$ & $0.000591^{\mathrm{c}}$ & $0.018955^{\mathrm{de}}$ & $0.035467^{f}$ & $2.229564^{\mathrm{d}}$ \\
\hline Ctrl * spray & $28.630^{\mathrm{f}}$ & $0.00093^{i}$ & $0.000441^{\mathrm{h}}$ & $0.000252^{\mathrm{d}}$ & $0.000499^{\mathrm{d}}$ & $0.013236^{\mathrm{f}}$ & $0.024158^{g}$ & $1.950168^{\mathrm{e}}$ \\
\hline Ctrl * standard & $39.372^{\mathrm{de}}$ & $0.00110^{\mathrm{h}}$ & $0.00048^{\mathrm{h}}$ & $0.00036^{\mathrm{c}}$ & $0.0052^{\mathrm{d}}$ & $0.01378^{\mathrm{ef}}$ & $0.03093^{f}$ & $1.95512^{\mathrm{d}}$ \\
\hline
\end{tabular}

Means in a column followed by the same letter are not significantly different at $5 \%$ level; results are the mean of three replicates.

Table 6 Effect of the double interaction between hormone*measuring time double interaction on studied traits of carnation.

\begin{tabular}{|c|c|c|c|c|c|c|c|}
\hline (Hormone*Measuring time) & MSI & TChl & Chla & Chlb & TSS & CAT & Pro \\
\hline SA50 $\mu \mathrm{M} * 1^{\text {st }}$ time & $61.8100^{\mathrm{ab}}$ & $0.00184^{\mathrm{bc}}$ & $0.000873^{\mathrm{de}}$ & $0.000514^{\mathrm{b}}$ & $0.000825^{\mathrm{a}}$ & $0.03028^{\mathrm{de}}$ & $4.5949^{\mathrm{bc}}$ \\
\hline SA50 $\mu M * 2^{\text {nd }}$ time & $50.6633^{\mathrm{bc}}$ & $0.00168^{\mathrm{cd}}$ & $0.000823^{\text {ef }}$ & $0.000472^{\mathrm{bc}}$ & $0.000796^{\mathrm{ab}}$ & $0.03101^{\mathrm{de}}$ & $3.9299^{\mathrm{ef}}$ \\
\hline SA50 $\mu \mathrm{M} * 3^{\text {rd }}$ time & $46.6083^{c}$ & $0.00147^{\mathrm{e}}$ & $0.000791^{\mathrm{efg}}$ & $0.000285^{\mathrm{h}}$ & $0.000593^{\text {def }}$ & $0.02786^{\mathrm{def}}$ & $3.9002^{\mathrm{ef}}$ \\
\hline SA100 $\mu M * 1^{\text {st }}$ time & $64.8867^{\mathrm{a}}$ & $0.00207^{\mathrm{ab}}$ & $0.001086^{\mathrm{a}}$ & $0.000591^{\mathrm{a}}$ & $0.00084^{\mathrm{a}}$ & $0.054845^{\mathrm{a}}$ & $4.9021^{\mathrm{a}}$ \\
\hline SA100 $\mu M * 2^{\text {nd }}$ time & $55.0667^{\mathrm{b}}$ & $0.00186^{\mathrm{bc}}$ & $0.001006^{\mathrm{abc}}$ & $0.000585^{\mathrm{a}}$ & $0.000803^{\mathrm{ab}}$ & $0.0443^{\mathrm{b}}$ & $4.7575^{\mathrm{ab}}$ \\
\hline SA100 $\mu M * 3^{\text {rd }}$ time & $49.7370^{\text {cde }}$ & $0.00169^{\mathrm{cd}}$ & $0.000967^{\text {bc }}$ & $0.000439^{\mathrm{bcd}}$ & $0.000551^{\mathrm{fg}}$ & $0.04295^{\mathrm{bc}}$ & $3.912345^{\mathrm{ef}}$ \\
\hline SA200 $\mu M * 1^{\text {st }}$ time & $57.8600^{\mathrm{b}}$ & $0.00152^{\mathrm{d}}$ & $0.000731^{\text {fghi }}$ & $0.000458^{\mathrm{bcd}}$ & $0.000701^{\mathrm{cd}}$ & $0.021981^{\mathrm{efg}}$ & $4.36244^{\mathrm{cd}}$ \\
\hline SA200 $\mu M * 2^{\text {nd }}$ time & $43.8280^{\text {cd }}$ & $0.00129^{\mathrm{g}}$ & $0.000642^{\text {hi }}$ & $0.000416^{\text {cde }}$ & $0.000633^{\text {cde }}$ & $0.021848^{\mathrm{efg}}$ & $4.193794^{\text {cde }}$ \\
\hline SA200 $\mu \mathrm{M} * 3^{\text {rd }}$ time & $35.4583^{\mathrm{d}}$ & $0.00092^{\mathrm{h}}$ & $0.000466^{1}$ & $0.000239^{\text {hij }}$ & $0.000459^{\mathrm{hi}}$ & $0.022104^{\mathrm{efg}}$ & $4.197395^{\text {cde }}$ \\
\hline BA50 $\mu \mathrm{M} * 1^{\text {st }}$ time & $57.1383^{b}$ & $0.00170^{\mathrm{cd}}$ & $0.00089^{\mathrm{de}}$ & $0.000516^{\mathrm{b}}$ & $0.000746^{\mathrm{c}}$ & $0.023508^{\mathrm{efg}}$ & $4.011608^{\mathrm{ef}}$ \\
\hline BA50 $\mu M * 2^{\text {nd }}$ time & $42.5617^{\text {cd }}$ & $0.00155^{\mathrm{d}}$ & $0.000801^{\mathrm{efg}}$ & $0.000481^{\mathrm{bc}}$ & $0.000655^{\text {cde }}$ & $0.023436^{\mathrm{efg}}$ & $3.087849^{\text {hi }}$ \\
\hline BA50 $\mu M * 3^{\text {rd }}$ time & $34.0817^{\mathrm{d}}$ & $0.00131^{\mathrm{f}}$ & $0.000753^{\mathrm{fgh}}$ & $0.000338^{\mathrm{fg}}$ & $0.000485^{\mathrm{h}}$ & $0.022588^{\mathrm{efg}}$ & $2.550495^{\mathrm{jkl}}$ \\
\hline BA100 $\mu M * 1^{\text {st }}$ time & $60.2833^{\mathrm{ab}}$ & $0.00212^{\mathrm{a}}$ & $0.001045^{\mathrm{ab}}$ & $0.000597^{\mathrm{a}}$ & $0.000747^{\mathrm{c}}$ & $0.03566^{\mathrm{d}}$ & $4.20515^{\text {cde }}$ \\
\hline BA100 $\mu M * 2^{\text {nd }}$ time & $47.7433^{c}$ & $0.00196^{\mathrm{b}}$ & $0.000941^{\mathrm{bcd}}$ & $0.000515^{\mathrm{b}}$ & $0.000641^{\text {cde }}$ & $0.028069^{\mathrm{def}}$ & $3.7409^{\mathrm{efg}}$ \\
\hline BA100 $\mu \mathrm{M} * 3^{\text {rd }}$ time & $41.3600^{\text {cd }}$ & $0.00170^{\mathrm{cd}}$ & $0.000897^{\mathrm{de}}$ & $0.000431^{\mathrm{bcd}}$ & $0.000462^{\mathrm{hi}}$ & $0.0299^{\mathrm{de}}$ & $3.2645^{\mathrm{h}}$ \\
\hline BA150 $\mu M * 1^{\text {st }}$ time & $53.0200^{\mathrm{bc}}$ & $0.00152^{\mathrm{d}}$ & $0.000713^{\text {fghi }}$ & $0.000454^{\mathrm{bcd}}$ & $0.000665^{\text {cde }}$ & $0.01587^{\mathrm{hij}}$ & $2.8492^{\mathrm{ij}}$ \\
\hline BA150 $\mu M * 2^{\text {nd }}$ time & $36.0200^{\mathrm{d}}$ & $0.00132^{\mathrm{f}}$ & $0.000594^{\text {hij }}$ & $0.000446^{\mathrm{bcd}}$ & $0.000609^{\text {def }}$ & $0.017609^{\text {ghi }}$ & $2.6575^{\mathrm{jk}}$ \\
\hline BA150 $\mu M * 3^{\text {rd }}$ time & $27.7283^{\mathrm{e}}$ & $0.00097^{\mathrm{jk}}$ & $0.000568^{\mathrm{ijk}}$ & $0.000257^{\mathrm{hi}}$ & $0.000418^{\mathrm{ij}}$ & $0.019126^{\mathrm{gh}}$ & $1.979^{\mathrm{m}}$ \\
\hline Ctrl * 1sttime & $47.7230^{\mathrm{c}}$ & $0.00127^{\text {ghi }}$ & $0.000632^{\text {hi }}$ & $0.000359^{\mathrm{f}}$ & $0.000603^{\text {def }}$ & $0.01325^{\text {hijk }}$ & $1.8815^{\mathrm{m}}$ \\
\hline Ctrl * 2ndtime & $31.3750^{\mathrm{de}}$ & $0.00102^{\mathrm{g}}$ & $0.000458^{1}$ & $0.000344^{\mathrm{fg}}$ & $0.000547^{\mathrm{fg}}$ & $0.01422^{\text {hijk }}$ & $2.9141^{\mathrm{ij}}$ \\
\hline Ctrl * 3rdtime & $22.9050^{\mathrm{e}}$ & $0.00076^{\mathrm{m}}$ & $0.000293^{\mathrm{m}}$ & $0.000224^{\mathrm{ijk}}$ & $0.000373^{\mathrm{jk}}$ & $0.01305^{\text {hijk }}$ & $1.0624^{\mathrm{n}}$ \\
\hline
\end{tabular}

Means in a column followed by the same letter are not significantly different at $5 \%$ level; results are the mean of three replicates. 
Table 7 Effect of the double interaction between measuring time * cultivar double interaction on studied traits of carnation.

\begin{tabular}{|lc|ccccc|c|c|}
$\begin{array}{l}\text { Double interaction } \\
\text { (measuring time*cultivar) }\end{array}$ & MSI & TChl & Chla & Chlb & TSS & CAT & POD & Pro \\
\hline $\mathbf{1}^{\text {st }}$ time * spray & $52.84^{\mathrm{b}}$ & $0.001668^{\mathrm{ab}}$ & $0.0007^{\mathrm{c}}$ & $0.000474^{\mathrm{b}}$ & $0.000692^{\mathrm{b}}$ & $0.0268^{\mathrm{ab}}$ & $0.05215^{\mathrm{a}}$ & $3.4745^{\mathrm{b}}$ \\
\hline $\mathbf{1}^{\mathrm{st}}$ time $^{*}$ standard & $62.22^{\mathrm{a}}$ & $0.001769^{\mathrm{a}}$ & $0.001^{\mathrm{a}}$ & $0.000555^{\mathrm{a}}$ & $0.000773^{\mathrm{a}}$ & $0.0294^{\mathrm{a}}$ & $0.05219^{\mathrm{a}}$ & $3.8610^{\mathrm{a}}$ \\
\hline $\mathbf{2}^{\text {nd }}$ time $^{*}$ spray & $46.75^{\mathrm{c}}$ & $0.001445^{\mathrm{b}}$ & $0.00081^{\mathrm{b}}$ & $0.000378^{\mathrm{c}}$ & $0.000647^{\mathrm{bc}}$ & $0.0290^{\mathrm{a}}$ & $0.03652^{\mathrm{c}}$ & $3.249^{\mathrm{c}}$ \\
\hline $\mathbf{2}^{\text {nd }}$ time $^{*}$ standard & $41.04^{\mathrm{d}}$ & $0.001607^{\mathrm{ab}}$ & $0.000647^{\mathrm{d}}$ & $0.000521^{\mathrm{a}}$ & $0.000691^{\mathrm{b}}$ & $0.0242^{\mathrm{b}}$ & $0.05009^{\mathrm{ab}}$ & $3.446^{\mathrm{bc}}$ \\
\hline $\mathbf{3}^{\text {rd }}$ time $^{*}$ spray & $33.27^{\mathrm{e}}$ & $0.001001^{\mathrm{c}}$ & $0.000657^{\mathrm{d}}$ & $0.000235^{\mathrm{d}}$ & $0.000401^{\mathrm{d}}$ & $0.0216^{\mathrm{c}}$ & $0.02840^{\mathrm{d}}$ & $3.054^{\mathrm{d}}$ \\
\hline $\mathbf{3}^{\text {rd }}$ time $^{*}$ standard & $40.41^{\mathrm{d}}$ & $0.001516^{\mathrm{b}}$ & $0.000742^{\mathrm{c}}$ & $0.000397^{\mathrm{c}}$ & $0.000554^{\mathrm{c}}$ & $0.0270^{\mathrm{ab}}$ & $0.04464^{\mathrm{b}}$ & $3.759^{\mathrm{ab}}$ \\
\hline
\end{tabular}

Means in a column followed by the same letter are not significantly different at $5 \%$ level; results are the mean of three replicates.

\subsection{Cultivar factor}

Results of cultivar factor study revealed that cultivars reaction to treatments in measured traits were significantly different with cultivars $(\mathrm{p} \leq 0.01)$. Generally standard cultivar gained the higher value in all the traits except chlorophyll a content, which was reported higher in the spray cultivar (table 4).

\subsection{Interaction effects}

Results of study showed that membrane stability index, chlorophyll a, b, total chlorophyll, sugar content, catalase, peroxidase enzymes activity and proline content were affected by double interaction (spraying hormones $\times$ cultivars) $(p \leq 0.01)$. Among these, double interaction of salicylic acid 100 $\mu \mathrm{M} \times$ standard carnations, got the highest values in membrane stability index, total chlorophyll, chlorophyll a, total solvable sugar content, catalase, peroxidase enzymes activity and proline content while interaction of benzyl adenine $100 \mu \mathrm{M} \times$ standard carnations improved the value of chlorophyll $b$ content only (table 5).

Similarly, double interaction of spraying hormones and time caused changes in all of considered traits except peroxidase enzyme activity. Interaction of salicylic acid $100 \mu \mathrm{M} \times 1$ st time of measurement made the highest values in membrane stability index, chlorophyll a, total solvable sugar content, catalase enzyme activity and proline content. Further, chlorophyll b content, and total chlorophyll content were reported higher in the interaction of benzyl adenine $100 \mu \mathrm{M} \times$ 1st time (table 6).

Also double interaction of time and cultivars had significant influence on all the tested parameters $(\mathrm{p} \leq 0.01)$ and the highest values of studied traits were related to interaction of $1^{\text {st }}$ time of measurement $\times$ standard carnation (tab 7).

Triple interaction (spraying hormones $\times$ time $\times$ cultivars) also had an influence on chlorophyll a, b and total chlorophyll, sugar content, catalase enzyme activity, and proline content $(p \leq 0.01)$. The highest values of studied trait affected by triple interaction (spraying hormones $\times$ timex cultivars) including total chlorophyll and chlorophyll $b$ content under influence of benzyl adenine $100 \mu \mathrm{M} \times 1$ st time of measurement $\times$ standard carnation while the value of chlorophyll a content, Total solvable sugar content, catalase enzyme activity, and proline content were higher under the influence of salicylic acid 100 $\mu \mathrm{M} \times 1$ st time of measurement $\times$ standard carnation. (table 8 ).

\section{Discussions}

The results of this research are coordinated with other published reports. According to Hamidimoghadam et al. (2014) studied effects of nano-silver, gibberellic acid and benzyl adenine on some postharvest quality characteristics and vase life of 'Pinkcastellaro' carnation cut flowers. They indicated that singular applying of GA3, NS, and BA showed a better influence on quality and vase life of 'Pinkcastellaro' carnation cut flower. Asil \& Karimi (2010) applied Benzyl adenine $\left(0,25,50\right.$ or $\left.75 \mathrm{mg} \mathrm{L}^{-1}\right)$ for improving quality and vase life of cut Eustoma flowers. It has been reported that all mentioned concentration of benzyladenine prolonged the vase life, although, spraying $25,50 \mathrm{mgL}^{-1}$ benzyladenine were more effective on extending vase life of cut Eustoma flowers, decreasing ethylelne production, reducing leaves chlorophyll and anthocyanin degradation beside that positive effects of salicylic acid on retarding flower senescence was represented (Ezhilmathi et al., 2007; Hatamzadeh et al., 2012).

Ataii et al. (2015) reported that spraying of salicylic acid delaying the postharvest senescence of Lisianthus cut flowers. They reported that this spraying increasing the catalase and ascorbate peroxidase activities and reducing lipoxygenase (LOX) enzyme activity as a factor for peroxiding lipids of membrane, vase life could be extended and flower senescence could be retarded via preserving membrane integrity by salicylic treatment. Further, Hatamzadeh et al. (2012) reported that Vase solution of $150 \mathrm{mg} / \mathrm{L}$ salicylic acid has positive influence in vase life of Gladiolus cut spikes by retarding fresh weight loss and lipid peroxidation and enrich the antioxidant activity of Peroxidase. Furthermore various studies recognized the effects of drought stress, salicylic acid and jasmonic acid on Hill reaction, chlorophyll a, chlorophyll b, chlorophyll $\mathrm{a}+\mathrm{b}$, -carotene and Xanthophyll were significant on Dracocephalum moldavica $\mathrm{L}$. 
Table 8 Effect of the triple interaction between hormone*measuring time * cultivar double on various studied traits of carnation.

\begin{tabular}{|c|c|c|c|c|c|c|}
\hline Triple interaction & TChl & Chla & Chlb & TSS & CAT & Pro \\
\hline SA $50 \mu M * 1$ st time $*$ spray & $0.00187^{\text {cd }}$ & $0.00106^{\mathrm{bc}}$ & $0.00051^{\text {hij }}$ & $0.00080^{\mathrm{def}}$ & $0.0308^{\mathrm{ij}}$ & $4.8450^{\mathrm{b}}$ \\
\hline SA $50 \mu M * 1^{\text {st }}$ time $*$ standard & $0.00181^{\mathrm{def}}$ & $0.00068^{\mathrm{kl}}$ & $0.00052^{\mathrm{hi}}$ & $0.00086^{\mathrm{bc}}$ & $0.0298^{\mathrm{ijk}}$ & $4.3448^{\mathrm{cd}}$ \\
\hline SA $50 \mu M * 2^{\text {nd }}$ time $*$ spray & $0.00160^{\mathrm{hi}}$ & $0.00095^{\mathrm{def}}$ & $0.00038^{\mathrm{p}}$ & $0.00078^{\text {defg }}$ & $0.0295^{\mathrm{ijk}}$ & $3.9438^{\text {cde }}$ \\
\hline SA $50 \mu M * 2^{\text {nd }}$ time $*$ standard & $0.00175^{\mathrm{fg}}$ & $0.00064^{\operatorname{lm}}$ & $0.00056^{\mathrm{de}}$ & $0.00081^{\mathrm{de}}$ & $0.0326^{\mathrm{i}}$ & $3.9161^{\text {cde }}$ \\
\hline SA $50 \mu M * 3^{\text {rd }}$ time $*$ spray & $0.00113^{\mathrm{qrs}}$ & $0.00078^{\text {hij }}$ & $0.00025^{\mathrm{w}}$ & $0.00043^{\text {rst }}$ & $0.0282^{\mathrm{kl}}$ & $3.8035^{\text {def }}$ \\
\hline SA $50 \mu M * 3^{\text {rd }}$ time $*$ standard & $0.00181^{\mathrm{def}}$ & $0.00087^{\text {fgh }}$ & $0.00032^{\mathrm{st}}$ & $0.0008^{\text {fgh }}$ & $0.0276^{\mathrm{klm}}$ & $3.9969^{\text {cde }}$ \\
\hline SA $100 \mu M * 1^{\text {st }}$ time $*$ spray & $0.002067^{\mathrm{b}}$ & $0.00099^{\text {cde }}$ & $0.00060^{\mathrm{ab}}$ & $0.00075^{\text {fghi }}$ & $0.0530^{\mathrm{b}}$ & $5.1715^{\mathrm{a}}$ \\
\hline SA $100 \mu M * 1^{\text {st }}$ time $*$ standard & $0.002078^{\mathrm{b}}$ & $0.00117^{\mathrm{a}}$ & $0.00057^{\mathrm{bcd}}$ & $0.00094^{\mathrm{a}}$ & $0.0567^{\mathrm{a}}$ & $5.2208^{\mathrm{a}}$ \\
\hline SA $100 \mu M * 2^{\text {nd }}$ time $*$ spray & $0.00176^{\mathrm{fg}}$ & $0.00113^{\mathrm{ab}}$ & $0.00058^{\mathrm{bc}}$ & $0.00073^{\text {ghi }}$ & $0.04743^{\mathrm{d}}$ & $4.2943^{\mathrm{cd}}$ \\
\hline SA $100 \mu M * 2^{\text {nd }}$ time $*$ standard & $0.00197^{\mathrm{bc}}$ & $0.00081^{\text {hij }}$ & $0.00061^{\mathrm{ab}}$ & $0.00088^{\mathrm{b}}$ & $0.04117^{\mathrm{ef}}$ & $4.6328^{\mathrm{bc}}$ \\
\hline SA $100 \mu M * 3^{\text {rd }}$ time $*$ spray & $0.00138^{\operatorname{lmn}}$ & $0.00104^{\text {bcd }}$ & $0.00037^{\mathrm{pq}}$ & $0.00045^{\mathrm{rs}}$ & $0.03815^{\mathrm{g}}$ & $3.5375^{\text {defg }}$ \\
\hline SA $100 \mu \mathrm{M} * 3^{\text {rd }}$ time $*$ standard & $0.00199^{\mathrm{bc}}$ & $0.00097^{\mathrm{def}}$ & $0.00051^{\text {hij }}$ & $0.00066^{\mathrm{jk}}$ & $0.04776^{\mathrm{d}}$ & $4.2874^{\mathrm{cd}}$ \\
\hline SA $200 \mu M * 1^{\text {st }}$ time $*$ spray & $0.00159^{\mathrm{hi}}$ & $0.00098^{\mathrm{def}}$ & $0.00041^{\mathrm{no}}$ & $0.00068^{\mathrm{j}}$ & $0.02515^{\mathrm{mn}}$ & $4.5776^{\mathrm{bc}}$ \\
\hline SA $200 \mu M * 11^{\text {st }}$ time $*$ standard & $0.00144^{\mathrm{kl}}$ & $0.00049^{\mathrm{pq}}$ & $0.00051^{\mathrm{hij}}$ & $0.00072^{\text {hi }}$ & $0.01881^{\text {opqr }}$ & $4.1474^{\mathrm{cd}}$ \\
\hline SA $200 \mu M * 2^{\text {nd }}$ time $*$ spray & $0.00123^{\mathrm{opq}}$ & $0.00068^{\mathrm{kl}}$ & $0.00033^{\mathrm{s}}$ & $0.00063^{\mathrm{jk}}$ & $0.02258^{\mathrm{no}}$ & $3.6984^{\text {def }}$ \\
\hline SA $200 \mu \mathrm{M} * 2^{\text {nd }}$ time $*$ standard & $0.00135^{\mathrm{mno}}$ & $0.00060^{\operatorname{lmn}}$ & $0.00050^{\mathrm{ijk}}$ & $0.00064^{\mathrm{jk}}$ & $0.02112^{\mathrm{op}}$ & $4.6893^{\mathrm{bc}}$ \\
\hline SA $200 \mu M * 3^{\text {rd }}$ time $*$ spray & $0.00078^{\mathrm{w}}$ & $0.00052^{\mathrm{op}}$ & $0.00017^{\mathrm{y}}$ & $0.00039^{\text {tuv }}$ & $0.02000^{\text {opq }}$ & $3.5676^{\text {defg }}$ \\
\hline SA $200 \mu \mathrm{M} * 3^{\text {rd }}$ time $*$ standard & $0.00106^{\mathrm{rt}}$ & $0.00041^{\mathrm{qr}}$ & $0.00031^{\text {stu }}$ & $0.00053^{\text {nop }}$ & $0.02421^{\mathrm{mno}}$ & $4.8273^{\mathrm{b}}$ \\
\hline BA $50 \mu M * 1^{\text {st }}$ time $*$ spray & $0.00173^{\mathrm{fg}}$ & $0.00101^{\text {cde }}$ & $0.00048^{\mathrm{kl}}$ & $0.00072^{\mathrm{hi}}$ & $0.02318^{\mathrm{no}}$ & $4.2282^{\mathrm{cd}}$ \\
\hline BA $50 \mu \mathrm{M} * 1^{\text {st }}$ time $*$ standard & $0.00166^{\mathrm{fgh}}$ & $0.00077^{\text {hij }}$ & $0.00055^{\text {ef }}$ & $0.00077^{\mathrm{efg}}$ & $0.02384^{\mathrm{mno}}$ & $3.7951^{\text {def }}$ \\
\hline BA $50 \mu M * 2^{\text {nd }}$ time $*$ spray & $0.00141^{\mathrm{klm}}$ & $0.00084^{\text {hi }}$ & $0.00036^{\mathrm{qr}}$ & $0.00065^{\mathrm{jk}}$ & $0.02153^{\mathrm{op}}$ & $3.2834^{\mathrm{efg}}$ \\
\hline BA $50 \mu \mathrm{M} * 2^{\text {nd }}$ time $*$ standard & $0.00170^{\mathrm{fgh}}$ & $0.00067^{\mathrm{kl}}$ & $0.00060^{\mathrm{ab}}$ & $0.00066^{\mathrm{jk}}$ & $0.02535^{\mathrm{mn}}$ & $2.8923^{\text {ghi }}$ \\
\hline BA $50 \mu \mathrm{M} * 3^{\text {rd }}$ time $*$ spray & $0.00100^{\mathrm{stu}}$ & $0.00067^{\mathrm{kl}}$ & $0.00023^{x}$ & $0.00040^{\mathrm{tu}}$ & $0.01988^{\mathrm{opq}}$ & $2.1432^{\mathrm{kl}}$ \\
\hline BA $50 \mu \mathrm{M} * 3^{\text {rd }}$ time $*$ standard & $0.00161^{\mathrm{hi}}$ & $0.00093^{\mathrm{def}}$ & $0.00045^{\mathrm{m}}$ & $0.00057^{\mathrm{mn}}$ & $0.02529^{\mathrm{mn}}$ & $2.9578^{\text {ghi }}$ \\
\hline BA $100 \mu \mathrm{M} * 1^{\text {st }}$ time $*$ spray & $0.00199^{\mathrm{bc}}$ & $0.00113^{\mathrm{ab}}$ & $0.00060^{\mathrm{ab}}$ & $0.00066^{\mathrm{jk}}$ & $0.02049^{\mathrm{opq}}$ & $3.4011^{\text {efg }}$ \\
\hline BA $100 \mu \mathrm{M} * 1^{\text {st }}$ time $*$ standard & $0.00224^{\mathrm{a}}$ & $0.00096^{\text {def }}$ & $0.00062^{\mathrm{a}}$ & $0.00083^{\text {cd }}$ & $0.05084^{\mathrm{bc}}$ & $3.1279^{\text {efgh }}$ \\
\hline BA $100 \mu \mathrm{M} * 2^{\text {nd }}$ time $*$ spray & $0.00207^{\mathrm{b}}$ & $0.00108^{\mathrm{bc}}$ & $0.00041^{\text {no }}$ & $0.00061^{\mathrm{jkl}}$ & $0.01931^{\text {opqr }}$ & $2.8294^{\text {ghi }}$ \\
\hline BA $100 \mu \mathrm{M} * 2^{\text {nd }}$ time $*$ standard & $0.00184^{\text {cde }}$ & $0.00072^{\mathrm{jk}}$ & $0.00060^{\mathrm{ab}}$ & $0.00068^{\mathrm{j}}$ & $0.03684^{\mathrm{gh}}$ & $4.6524^{\mathrm{bc}}$ \\
\hline BA $100 \mu M * 3^{\text {rd }}$ time $*$ spray & $0.00155^{\text {hij }}$ & $0.00094^{\mathrm{def}}$ & $0.00032^{\mathrm{st}}$ & $0.00042^{\text {rst }}$ & $0.01814^{\mathrm{pqr}}$ & $3.6901^{\text {def }}$ \\
\hline BA $100 \mu \mathrm{M} * 3^{\text {rd }}$ time $*$ standard & $0.00184^{\text {cde }}$ & $0.00095^{\mathrm{def}}$ & $0.00054^{\mathrm{efg}}$ & $0.00050^{\mathrm{pq}}$ & $0.04172^{\mathrm{e}}$ & $4.7202^{\mathrm{bc}}$ \\
\hline BA $150 \mu \mathrm{M} * 1^{\text {st }}$ time $*$ spray & $0.00150^{\mathrm{ijkl}}$ & $0.00089^{\mathrm{fg}}$ & $0.00037^{\mathrm{pq}}$ & $0.00065^{\mathrm{jk}}$ & $0.01671^{\mathrm{rs}}$ & $2.8442^{\text {ghi }}$ \\
\hline BA $150 \mu \mathrm{M} * 1^{\text {st }}$ time $*$ standard & $0.00153^{\mathrm{ijk}}$ & $0.00053^{\mathrm{op}}$ & $0.00053^{\text {fgh }}$ & $0.00068^{\mathrm{j}}$ & $0.01503^{\text {rstu }}$ & $2.4709^{\mathrm{jk}}$ \\
\hline BA $150 \mu M * 2^{\text {nd }}$ time $*$ spray & $0.00112^{\mathrm{qrs}}$ & $0.00056^{\mathrm{no}}$ & $0.00032^{\mathrm{st}}$ & $0.00059^{\mathrm{klm}}$ & $0.01612^{\text {rst }}$ & $2.1372^{\mathrm{kl}}$ \\
\hline BA $150 \mu M * 2^{\text {nd }}$ time $*$ standard & $0.00153^{\mathrm{ijk}}$ & $0.00063^{\operatorname{lm}}$ & $0.00058^{\mathrm{bc}}$ & $0.00063^{\mathrm{jkl}}$ & $0.01911^{\text {opqr }}$ & $1.8207^{\mathrm{klmn}}$ \\
\hline BA $150 \mu \mathrm{M} * 3^{\text {rd }}$ time $*$ spray & $0.00068^{\mathrm{x}}$ & $0.00045^{\mathrm{pq}}$ & $0.00016^{\mathrm{yz}}$ & $0.00037^{\mathrm{uv}}$ & $0.01552^{\text {rstu }}$ & $3.3013^{\text {efg }}$ \\
\hline BA $150 \mu \mathrm{M} * 3^{\text {rd }}$ time $*$ standard & $0.00126^{\mathrm{op}}$ & $0.00069^{\mathrm{kl}}$ & $0.00035^{\mathrm{qrs}}$ & $0.00047^{\mathrm{r}}$ & $0.02274^{\text {no }}$ & $2.3971^{\mathrm{jk}}$ \\
\hline Ctrl $* 1^{\text {st }}$ time $*$ spray & $0.00139^{\operatorname{lmn}}$ & $0.00079^{\text {hij }}$ & $0.00035^{\mathrm{rs}}$ & $0.00059^{\mathrm{klm}}$ & $0.01495^{\text {rstu }}$ & $1.9600^{\mathrm{klm}}$ \\
\hline Ctrl $* 1^{\text {st }}$ time $*$ standard & $0.00173^{\mathrm{fg}}$ & $0.00101^{\text {cde }}$ & $0.00038^{\mathrm{pq}}$ & $0.00062^{\mathrm{jkl}}$ & $0.01155^{\mathrm{vw}}$ & $1.8029^{\mathrm{klmn}}$ \\
\hline Ctrl $* 2^{\text {nd }}$ time $*$ spray & $0.00166^{\mathrm{fgh}}$ & $0.00077^{\text {hij }}$ & $0.00027^{\mathrm{v}}$ & $0.00055^{\text {no }}$ & $0.01324^{\mathrm{uv}}$ & $1.1912^{\circ}$ \\
\hline Ctrl $* 2^{\text {nd }}$ time $*$ standard & $0.00141^{\mathrm{klm}}$ & $0.00084^{\text {hi }}$ & $0.00042^{\mathrm{n}}$ & $0.00055^{\text {no }}$ & $0.01287^{\mathrm{uv}}$ & $2.6994^{\text {hij }}$ \\
\hline Ctrl $* 3{ }^{\text {rd time }} *$ spray & $0.00170^{\mathrm{fgh}}$ & $0.00067^{\mathrm{kl}}$ & $0.00014^{z}$ & $0.00036^{\mathrm{uvw}}$ & $0.01152^{\mathrm{vw}}$ & $0.9337^{\mathrm{op}}$ \\
\hline Ctrl $* 3^{\text {rd }}$ time $*$ standard & $0.00100^{\mathrm{stu}}$ & $0.00067^{\mathrm{kl}}$ & $0.00031^{\text {stu }}$ & $0.00039^{\text {tuv }}$ & $0.01693^{\mathrm{rs}}$ & $3.1288^{\text {efgh }}$ \\
\hline
\end{tabular}

Means in a column followed by the same letter are not significantly different at $5 \%$ level; results are the mean of three replicates.

It was indicated the use of Jasmonic acid and salicylic acid increased antioxidant and non antioxidant defense is power (Abbaspour \& Rezaei, 2014). Another study revealed foliar application of salicylic acid (SA) and jasmonic acid (JA) significantly affected number of flower, diameter of flower, diameter of stalk, stalk length, fresh weight and total chlorophyll contents of rose (cv. 'Angelina'). About of using salicylic acid, the maximum number of flower, diameter of stalk and total chlorophyll contents reported at $14 \mathrm{ppm}$ and most of flower diameter, stalk length and fresh weight was measured in 21 ppm (Jahanbazi et al., 2014). 


\section{Conclusion}

There were progressive effects of plants hormones on studied traits in both of spray and standard carnations. Although, the plants hormones which were applied on standard carnations indicated much better effects than spray carnation, all measured traits improved. Results of this study showed that salicylic acid $100 \mu \mathrm{M}$, and benzyl adenine $100 \mu \mathrm{M}$ had more effects than others. Due to their ease of use and positive effects on morphological parameters, spraying of benzyl adenine and salicylic acid are one of the best methods in floriculture.

\section{Conflict of interest}

Authors would hereby like to declare that there is no conflict of interests that could possibly arise.

\section{References}

Abbaspour H, Rezaei H (2014) Effects of Salicylic acid and Jasmonic acid on Hill Reaction and Photosynthetic Pigment (Dracocephalum moldavica L.) in Different Levels of Drought Stress. International journal of Advanced Biological and Biomedical Research $2: 2850-2859$.

Arias BS, Lopez MJA (2010) Control of growth and development of ornamental plants, the Polytechnic University of Cartagena available on www.interempresas.net/Horticola/Articulos/45284-Controldel-crecimiento-y-desarrollo-de-plantas-ornamentales.html access on 26th November, 2015.

Arnon AN (1967) Method of extraction of chlorophyll in the plants. Agronomy Journal 23:112-121.

Ataii D, Naderi R, Khandan-Mirkohi A (2015) Delaying of Postharvest Senescence of Lisianthus Cut Flowers by Salicylic Acid Treatment. Journal of Ornamental Plants 5: 67-74.

Australian Government (2006) The Biology and Ecology of Dianthus caryophyllus L. (Carnation). Department of Health and Ageing Office of the Gene Technology Regular 1-22.

Bates LS, Waldren RP, Teare ID (1973) Rapid determination of free proline for water-stress studies. Plant and Soil 39: 205207. DOI: 10.1007/BF00018060.

Behroozian M, Jafari A, Farsi M (2013) RAPD analysis of genetic variation within and among natural populations of two species of Dianthus L. (Caryophyllaceae) in NE Iran. The Iranian Journal of Botany 19: 194-201.

Bubán T (2000) The use of benzyladenine in orchard fruit growing: a mini review. Plant Growth Regulation 32: 381-390. DOI: $10.1023 / \mathrm{A}: 1010785604339$
Dey PM (1990) Methods in plant biochemistry. Vol-II. Carbohydrates. (Publ.) Academic Press London.

Ezhilmathi K, Singh VP, Arora A, Sairam RK (2007) Effect of 5 -sulfosalicylic acid on antioxidant activity in relation to vase life of Gladiolus cut flowers. Plant Growth Regulation 51: 99108. DOI: $10.1007 / \mathrm{s} 10725-006-9142-2$

Hamidimoghadam E, Rabiei V, Nabigol A, Farrokhi J (2014) Postharvest Quality Improvement of Carnation (Dianthus caryophyllus L.) Cut Flowers by Gibberellic Acid, Benzyl Adenine and Nano Silver. Agricultural Communications 2: 2834.

Asil MH, Karimi M (2010) Efficiency of Benzyladenine reduced ethylene production and extended vase life of cut Eustoma flowers. Plant Omics Journal 3:199-203.

Hatamzadeh A, Hatami M, Ghasemnezhad M (2012) Efficiency of salicylic acid delay petal senescence and extended quality of cut spikes of Gladiolus grandiflora cv 'wing's sensation'. African Journal of Agricultural Research 7: 540-545.DOI: http://dx.doi.org/10.5897/ajar11.962

Isinger W (1977) Role of cytokinins in carnation flower senescence. Plant Physiolology 59, 707-709. DOI: http://dx. doi.org/10.1104/pp.59.4.707

Jahanbazi T, Mortezaienejad F, Jafararpoor M (2014) Impact of salicylic acid and jasmonic acid on keeping quality of rose (cv. 'Angelina') flower. Journal of Novel Applied Sciences 3 : 1328-1335.

John Carey D, Mark K (2008) the Effects of Benzyladenine on Ornamental Crops. A thesis submitted to the Graduate Faculty of North Carolina State University in partial fulfillment of the requirements for the Degree of Master of Science. Raleigh, North Carolina

Jurgen A, Witt T, Gottrsberger G (2003) flower scent composition in Dianthus and Saponaria species. Biological Systematic and Ecology. 31: 345-357. DOI: http://dx.doi.org/10.1016/s0305-1978(02)00173-4

Khademi Z, Ershadi A (2013) Postharvest application of salicylic acid improves torability of peach (Prunus persica cv. Elberta) Fruits. International Journal of Agriculture and crop Sciences. 5: 651-655.

Kharrazi M, Nemati H, Tehranifar A, Bagheri A, Sharifi A (2011) In Vitro Culture of Carnation (Dianthus caryophyllus L.) Focusing on the Problem of Vitrification. Journal of Biodiversity and Environmental Sciences 5: 1-6.

OGTR (2006) The biology and ecology of Dianthus caryophyllus L. (Carnation). Australian Government. Department of health and ageing, Office of the Gene Technology Regulator. 
Ramtin A, Kalatejari S, Naderi R, Matinizadeh M (2015) Effect of Pre-harvest Foliar Application of Benzyl adenine and Salicylic acid on Carnation cv. Spray and Standard. Biological Forum - An International Journal 7: 955-958

Rani P, Meena Unni K, Karthikeyan J (2004) Evaluation of antioxidant properties of berries. Indian journal of Clinical Biochemistry 19 :103-110. DOI: 10.1007/BF02894266.

Sairam RK (1994) Effect of moisture stress on physiological activities of two contrasting wheat genotypes. Indian Journal of Experimental Biology 32:584-593.

Schmülling T (2004) Cytokinin. In: Lennarz W, Lane MD (Eds) Encyclopedia of Biological Chemistry. Academic Press/Elsevier Science.
Supapvanich S, Promyou S (2013) Efficiency of Salicylic Acid Application on Postharvest Perishable Crops. Springer Science+Business Media Dordrecht. _15. 339-355. DOI: 10.1007/978-94-007-64286

Yao H, Tian S (2005) Effects of pre- and post-harvest application of salicylic acid or methyl jasmonate on inducing disease resistance of sweet cherry fruit in storage. Postharvest Biology and Technology 35: 253-262.DOI: 10.1016/j.postharvbio.2004.09.001

Zarghami M, Shoor M, Ganjali A, Moshtagi N, Tehranifar A (2014) Effect of salicylic acid on morphological and ornamental characteristics of petunia hybrid at drought stress. Indian Journal of Fundamental and Applied Life Sciences 4 : 523-532 